\title{
Unusual Presentation of Guillain-Barré Syndrome following Traumatic Bone Injuries: Report of Two Cases
}

\author{
Jasem Yousef Al-Hashel John K. John Periasamy Vembu \\ Department of Neurology, Ibn Sina Hospital, Safat, Kuwait
}

\section{Key Words}

Guillain-Barré syndrome · Intravenous immunoglobulin . Critical illness polyneuropathy $\cdot$ Nerve conduction velocity

\begin{abstract}
Objective: To report two cases of Guillain-Barré syndrome (GBS) which occurred following traumatic bone injuries. Presentation and Intervention: Two patients presented with traumatic bone injuries. The first was a 47-year-old female who was admitted with fracture of both tibial bones sustained during a road traffic accident. One week after surgical fixation of the fracture, she developed areflexic weakness of all four limbs and respiratory muscle weakness. The nerve conduction study was consistent with GBS. She was administered intravenous immunoglobulins which was repeated after 2 weeks. She recovered gradually. The second patient was a 31-year-old male who was admitted with 4-days history of severe back pain which occurred when he lifted a heavy weight. He then developed ascending areflexic weakness of all four limbs and bifacial weakness. X-ray and magnetic resonance imaging of the lumbosacral spine revealed fracture of $L 1$ and $L 2$ vertebrae. Nerve conduction studies confirmed the diagnosis of GBS. He was given mechanical ventilatory support and was treated with intravenous immunoglobulins and later plasmapheresis. However, his condition gradually deteriorated as he developed aspiration pneumonia and sepsis with multi-organ failure and
\end{abstract}

finally expired. Conclusion: These cases highlight the importance of considering GBS as a differential diagnosis when patients with traumatic bone injuries develop acute neuromuscular weakness. Early diagnosis and treatment may prevent morbidity and mortality.

Copyright $\odot 2013$ S. Karger AG, Basel

\section{Introduction}

Guillain-Barré syndrome (GBS) often follows bacterial infections or viral infections like cytomegalovirus or herpes viruses [1]. It has also been reported in association with malignancies, cardiac surgery, renal transplant and spinal surgery $[2,3]$. GBS has rarely been reported to occur following post-traumatic bone injuries or after orthopaedic surgery [4-6]. We report 2 cases of GBS that occurred in the intensive care unit (ICU) in patients with either traumatic bone injuries or orthopaedic surgery.

\section{Case Reports}

Case 1

A 47-year-old female had a car accident and sustained fracture of both tibial bones. She underwent internal fixation of the fractured tibial bones under general anaesthesia. One week later she developed generalized muscle weakness and acute respiratory distress. She was transferred to the ICU where mechanical ventila-

\begin{tabular}{ll}
\hline KARGER & $\begin{array}{l}\text { Co 2013 S. Karger AG, Basel } \\
1011-7571 / 13 / 0226-0597 \$ 38.00 / 0\end{array}$ \\
E-Mail karger@karger.com & $\begin{array}{l}\text { This is an Open Access article licensed under the terms } \\
\text { of the Creative Commons Attribution-NonCommercial- } \\
\text { www.karger.com/mpp }\end{array}$ \\
& $\begin{array}{l}\text { NoDerivs 3.0 License (www.karger.com/OA-license), appli- } \\
\text { cable to the online version of the article only. Distribution } \\
\text { for non-commercial purposes only. }\end{array}$
\end{tabular}

Dr. Periasamy Vembu, MD, DM

Department of Neurology

Ibn Sina Hospital

PO Box 25427, Safat 13115 (Kuwait)

E Mail drvperiasamy@yahoo.com 
tory support was given after intubation. Clinically she had bilateral lower motor neuron facial palsy and quadriparesis. The upper limb power was $3 / 5$ bilaterally and lower limbs were $0 / 5$ proximally and 2/5 distally. She had total areflexia of all four limbs and the plantar responses were mute. She was assessed by a neurologist and a diagnosis of acute GBS was suspected. Nerve conduction study (NCS) showed prolonged distal latencies, absent $\mathrm{H}$ reflex and reduced conduction velocities suggestive of acute demyelinating polyradiculoneuropathy. The blink reflex was abnormal with prolonged motor latency of both facial nerves. The patient refused lumbar puncture, hence cerebrospinal fluid examination was not done; as she complained of back pain. Other investigations including full blood count, blood biochemistry, autoimmune screen, protein electrophoresis, and porphyria screening were negative. Serological tests for Mycoplasma pneumonia, Epstein-Barr virus and hepatitis virus were negative. The clinical picture and electrophysiological findings were consistent with GBS. She was treated with intravenous immunoglobulin (IVIG; $0.4 \mathrm{~g} / \mathrm{kg}$ for 5 days). While in the ICU she developed severe bulbar weakness. After the first course of IVIG, she started to improve slowly. The facial weakness and motor power in her limbs improved slowly. However, weaning from the ventilator was delayed due to bulbar weakness and inter-current respiratory tract infection. A second course of IVIG was given after 2 weeks. Chest infection was treated with piperacillin and tazobactam along with chest physiotherapy. Subsequently she recovered and after 3 weeks of ICU care, she was extubated. This patient improved well and was able to walk with minimum support and eventually discharged from the hospital.

\section{Case 2}

A 31-year-old male developed weakness of both lower limbs and severe low back pain after lifting a gas cylinder. Four days later he was admitted to Adan Hospital. X-ray of the lumbosacral spine revealed simple fracture at L1 and L2 vertebrae and magnetic resonance imaging of the lumbar spine confirmed the fracture. Within the next few days the weakness gradually progressed to $3 / 5$ power in the upper limbs and $0 / 5$ in the lower limbs bilaterally and bilateral lower motor neuron facial palsy. Deep tendon reflexes were absent in all four limbs and the plantar response was mute. He was assessed by a neurologist and was transferred to the ICU as he developed respiratory distress and bulbar weakness. NCS showed prolonged distal latencies with conduction block, prolonged $\mathrm{F}$ waves, reduced $\mathrm{M}$-wave amplitudes, reduced nerve conduction velocities and absent $\mathrm{H}$ reflexes. Blink reflex was abnormal with prolonged latencies and reduced amplitudes. Routine blood counts and blood biochemistry were normal. Connective tissue disease and porphyria screening were negative. Serological tests for M. pneumonia, Epstein-Barr virus and hepatitis virus were negative. Cerebrospinal fluid study was not done because the patient refused it as he had back pain. The clinical and electrophysiological features were consistent with GBS. He was treated with a course of IVIG $(0.4 \mathrm{~g} / \mathrm{kg} /$ day for 5 days $)$. While in the ICU he developed aspiration pneumonia and septicaemia which was treated by meropenem and ciprofloxacin. Follow-up electrophysiological studies showed progression of neuropathy with features of mixed axonal and demyelinating neuropathy. As he did not respond to the initial course of IVIG, he was treated with six sessions of plasmapheresis. However, he continued to be febrile in spite of treatment with appropriate antibiotics. He de- veloped cardiovascular instability and renal impairment. Twentyfive days later the patient expired after developing cardiac arrest and resuscitative measures failed. The probable cause of death was septicaemic shock with multi-organ failure secondary to septicaemia.

\section{Discussion}

The 2 cases reported here had rapidly evolving ascending paralysis which was preceded by fracture of bones. The diagnosis of GBS was made because both cases had typical history, clinical features and satisfied the electrophysiological criteria [7]. Even though it is rare, cases of GBS have been reported following spinal, cranial, gastrointestinal, genitourinary, maxillofacial, orthopaedic, pulmonary, eye and cardiac surgeries [4-6]. Both patients in our report were suspected to have developed GBS following trauma and fracture of the bones. In case 1 the clinical manifestations of GBS occurred more than 1 week after the trauma and about 1 week after the surgery. The duration of fracture vertebra and onset of illness was also within a week in case 2 . The interval between the time of trauma or surgery and the onset of symptoms of GBS is usually from a few days to a few weeks - the average being 1-2 weeks [6]. Several mechanisms have been suggested for development of GBS following surgery or traumatic bone injuries [8]. These mechanisms include missed viral infections, surgical stress, genetic susceptibility, anaesthetic drugs and antibacterial peptides which are generated following surgery (fig. 1). Major stress of surgery and trauma may alter both cellular and humoral immunities resulting in activation of latent processes that would in turn affect the immunological system. Such changes have been documented following spinal cord injury [8].

The standard treatment of GBS includes supportive care and immunotherapy, either IVIG or plasmapheresis, as was done for our 2 patients. Case 1 recovered but unfortunately case 2 expired because he developed fever and sepsis while in the ICU and progressed to multiorgan failure. In this patient the electrophysiological study done earlier was suggestive of demyelinating neuropathy consistent with GBS. However, he failed to respond to the initial course of IVIG. Whether the sepsis and multi-organ failure which are important in the pathogenesis of critical illness neuropathy (CIP) contributed to his late deterioration is debatable, but difficult to prove in the setting of demyelinating neuropathy. In such a situation, follow-up NCS demonstrating pro- 
Fig. 1. Probable mechanism of GBS following stress of surgery or trauma.

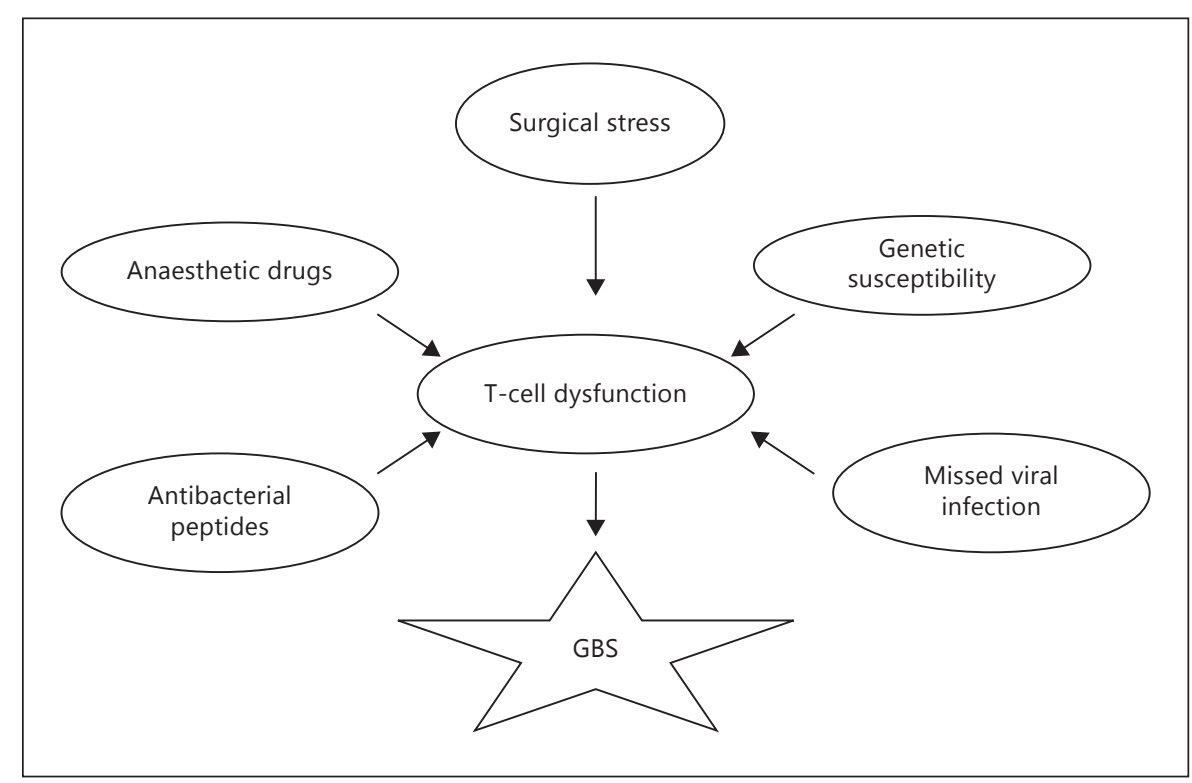

gressive axonal neuropathy is unlikely to be helpful as it can occur as a result of CIP or due to secondary axonal loss occurring in severe demyelination of GBS. In such a situation, treatment with IVIG is the best option if there is no contraindication for IVIG, as it is the standard treatment of GBS and also reported to be beneficial in CIP [9].

\section{Conclusion}

These cases highlight the importance of considering GBS as a differential diagnosis when patients with traumatic bone injuries develop acute neuromuscular weakness. Early diagnosis and treatment may prevent morbidity and mortality.

\section{References}

1 Hughes RA, Cornblath DR: Guillain-Barré syndrome. Lancet 2005;366:1653-1666.

$>2$ Vembu P, Al-Shubaili A, Al-Khuraibet A, et al: Guillain-Barré syndrome in a case of acute lymphoblastic leukemia, a case report. Med Princ Pract 2003;12:272-275.

3 Algahtani $\mathrm{H}$, Moulin DE, Bolton CF, et al: GBS following cardiac surgery. Difficult diagnosis in the intensive care unit. J Neurosci ( $\mathrm{Ri}$ yadh) 2009; 14:374-378.
-4 Riebel GD, Heller JG, Hopkins LC: GuillainBarré syndrome after an operation on the spine. J Bone Joint Surg Am 1995;77:15651567.

5 Jacka MJ: GBS following thoracic spinal cord trauma. Can J Anesth 2008;55:441-446.

$>6$ Lee MC, Campbell R, Bom C: GBS after failed pelvic fracture fixation. J Trauma 2009;67: E132-E135.

7 Asbury AK, Cornblath DR: Assessment of current diagnostic criteria for Guillain-Barré syndrome. Ann Neurol 1990;27(suppl):S21S24.
8 Ankeny DP, Phillip G: Mechanisms and implications of adaptive immune responses after traumatic spinal cord injury. Neuroscience 2009;158:1112-1121.

9 Mohr M, Englisch L, Roth A, et al: Effects of early treatment with immunoglobulin on critical illness polyneuropathy following multiple organ failure and Gram-negative sepsis. Intensive Care Med 1997;23:1144-1149. 